\title{
Scheinbare Gehirnzufälle
}

im Verlauf einer eitrigen Trommelhöhlenentzündung. Wegbleiben derselben nach einer einzigen Katheterisirung. Ein Beitrag zur klinischen Bedeutung des gesteigerten intraauriculären Drucks.

$$
\text { Von }
$$

Dr. Moos,

Privatdoeent in Heidelberg.

Der Güte des Herrn Professor Friedreich verdanke ich die Gelegenheit zur folgenden Beobachtung, den grössten Theil der Krankengeschichte dessen Assistenten, Herrn Ribstein.

Rosa Quadi, 30 Jahre alt, Wärterin auf der medizinischen Klinik, früher immer gesund, regelmässig menstruirt, bekam den 17. Dezember 1865 offer Frost, abweehselnd mit Hitze, verlor den Appetit, hatte viel Durst, 'Kopfschmerzen, war matt in den Gliedern und hatte etwas Husten. Stuhlgang seit einigen Tagen angehalten.

18. Dez. 120 Puls, 31,6 Temperatur.
19.

88

92
30,2
30,4
Morgens.
Abends.

Zunge belegt, kein Appetit, Kopfschmer», bald.Frösteln, bald Hitze, viel Durst, etwas Husten, Mattigkeit in den Gliedern. Innere Organe normal; nur auf den Lungen einzelne Rhonehi. Kuappe Diat. Zwei dreigränige Calomeldosen.

20. Dez. 84. Puls, 30 Temp. Morgens.

$$
96 \text { " } 30,7 n \text { Abends. }
$$

Kein Stuhl; Zunge belegt, kein Appetif, viel Durst. Ziemlich viel Husten mit eitrig schaumigem Auswurf; auf den Lungen ziemlich viel Rhonchi. Schmerz beim 
198 MOOS : Scheinb. Gehirnzufälle i. Verl. ein. eiter. Trommelhöhleneatzünd. etc.

Schingen. Rachen etwas geröthet, Tonsillen nich geschwollen, dagegen die Drüsen der Submaxillargegend. Priessnitz'scher Umschlag. Abends Brechneigung, öfteres Aufstossen. Potio Riveri.

21. Dez. Puls 100, Temp. 30,6 Morgens.

"92, $, 30,8$ Abends.

Befinden nnd Zustand ohngefüh wie gestern. Brechneigung verschwunden. Klysma.

22. Dez. Puls 84, Temp. 30,4 Morgens.

"96, $\quad 30,8$ Abends.

Ein Stnhlgang. Verminderung des Hustens and Abnahme der Rhonohi; keine Schlingbeschwerden.

23. Dez. Puls 104, Temp. 30,1 Morgens.

$88, \pi \quad 30,4$ Abends.

Kein Stuhl. Zunge belegt, wenig Appetit. Schmersen und Sausen im Ohr der rechten Stite, Kopfschmerz. Ordin.: Ol. hyoseyam. coct. 3ij, Tinctur. opii crocata guti. 10. Zum Einträufeln.

24. Dez. Puls 100, Temp. 30,2 Morgerıs.

"100, "30,3 Abends.

Kein Stuhl. Stat. id. Warme Umschlëge auf das rechte Ohr. Klysma.

25. Dez. Puls 88, Temp. 30. Morgens.

$$
\text { "100, "30,8 Abends. }
$$

Kein Stwhl. Abends Röthung und ziemlich starke Schmerzhaftigkeit am rechten Warzenfortsatz.

26. Dez. Puls 92, Temp. 30,5 Morgens.

$n$ 100, "31,0 Abends. Status idem.

Zwei Blutigel an den rechten Warzenfortsatz. Aqua laxativa. Darnach 3 Stühle.

27. Dez. Puls 96, Temp. 30,4 Morgens.

\#100, \#30,6 Abends.

Schmerzen im Obr geriager. Gebör Null. 4 stühle.

28. Dez. Puls 92, Temp. 30,4 Morgens.

"96, $, 30,4$ Abends.

Status, wie gestem. Zungembelag, kein Appetit, ein Stuhl.

29. Dez. Puls 84, Tromp. 29,8 Morgens.

$$
\text { "100, \#30,3 Abends. }
$$

Kein Stuhl. Erneuerte heftige Srhmerzen im rechten Ohre; starkes Sausen, Empfindlichkeit im Warsenfortsats. Orilin.: wie bisher, ausserdem 3 Blutigel an den Warzenfortsatz. Gegen Abond Nachlass der Schmerzen, Eiterausfluss aus dem Gchörgang, Ausspritzen nait warmem Wasser.

30. Dez Puls 104, Temp. 30,2 Morgens.

$$
\text { 92, "30,4 Abends. }
$$

Kein Stubl. Schmerzen und Ausfuss wie gestern Abend. Warzenfortsatz noch ziemlich empfindlich. Kein Stuhl.

31. Dez. Morgens. Puls 84, Temp. 30,2.

Drei Stuhlgänge. Schuindel, Brechneigung bis zu wirklichem Erbochen in der Nacht. Zunge rein, kein Appetit. Ausfluss aus dem Ohr und Schmerzen in demselben dauern fort. Abeads fühlt sich Patientin ziemlich wohl. Puls 92, Temp. 30,4.

Den 1. Januar 1866. Puls 84, Temp. 30,1 Morgens.

$$
\text { "88, \#30,6 Abeads. }
$$

Fin Stuhlgang, Zunge wenig belegt, kein Appetit, Schwindel, Brechneigung bis 
zu wirklichem Erbrechen in der Naeht. Ohrenfluss wie bisher, Ohrenschmerz gering. Während des Tags keine Breehneigung, kein Schwindel. Sonst Stat. idem Abends. Klysma.

2. Januar. Puls 80 , Temp. 29,4 Morgens. " 84, "30,2 Abends.

Heute Morgen wieder Uebelkeit, sonst Status idem. Kein Stubl.

3. Januar. Puls 84, Ternp. 29,4 Morgens.

$$
\text { " 88, } \Rightarrow 30,1 \text { Abends. }
$$

Ein Stuhl. Schmerz und Ausfluss in rechten Ohr geringer. Das Picken einer Taschenuhr wird bei directer Berïhrung gehört. Wenig Appetit.

4. Januar. Puls 84, Temp. 29,4 Morgens.

$$
\text { "84, } " 30,4 \text { Abends. }
$$

Nachts starke Schmerzen im rechten Ohr, heute Morgen Sausen. Ord. Klysma. Darauf Stuhlgang. Abends weder Schmerzen, noch Ausfluss.

5. Januar. Puls 108, Temp. 30,2 Morgeus.

$$
" 92, " 30,4 \text { Abends. }
$$

Nacht schmerzlos, wenig Sausen, kein Ausfuss. Appetit schleeht. In Laufe des Tages Erneuerung der Kopf- nnd Ohrenschmerzen, des Ausflusses. Uhr 11/2 Zoll.

6. Januar. Puls 88, Temp. 29,6 Morgens.

$$
\text { "90, "30 Abends. }
$$

Während der schlaflosen Nacht riel Ausfiuss and heftiger Kopfschmerz, wenig Ohrenschmerz. Sausen. Gegen Abend Steigerung der subjectiven Beschwerden. Ein Stuhl.

7. Januar. Puls 84, Temp. 29,6 Morgens.

$$
\text { " } 82, \quad 30,3 \text { Abends. }
$$

Ausfluss und Sausen continuirlich. Schmerzen im Obre geringer. Im Laufe des Tages sistirt der Ausfluss. Im Trommelfell wird eine Perforation constatirt.

8. Januar. Puls 100, Temp. 30,2 Morgens.

$$
" 88, " 30,6 \text { Abends. }
$$

Schmerzen im rechten Ohr, aber weniger Kopfechmerz und weniger Sausen. Ausfinss wieder vorhanden. Während der Nacht Debelleit und Erbrechen. Ein Stuhl.

Gegen Abend nimmt der Kopfschmerz reehts, vorzüglioh im Hinterkopf, wieder za. Schwindelgefühl während des Tags.

9. Januar, Puls 84, Temp. 30,2 Morgeas.

$$
" 100, \quad 30,1 \text { Abends. }
$$

Während der Nacht heftige Schmerzen im r. Ohr und auf der rechten Seite des Hinterkopfs, viel Ausfluss aus dem Ohr, starker Schwindel, Brechneigung; Zunge etwas belegt, kein Appetit, kein Stuhl. Während des Tags bis Abends kein Ausfuss. Klysma.

10. Januar. Puls 80 , Temp. 29,6 Morgens.

$$
\text { "92, "30,5 Abends. }
$$

Schlaf gut. Geringere Schmerzen und Sausen. Kein Ausfluss. Gegen Morgen Erbrechen, später Nachlass der Brechneigung. Kein Ausfluss. In den letzten Tagen häufig Gähnen. Im Laufe des Tages kein Ausfluss, starker Kopfschmerz, viel Schwindel und Brechneigung.

11. Januar. Puls 96, Temp. 29,5 Morgens. "92, "30,1 Abends.

Zunge rein, kein Appetit, kein Stuhl, kein Austluss. Schmerzen und Sausen im rechten $\mathrm{Ohr}$, weniger Kopfschmerz, aber Gefühl von Schwere, Schwindel, keine 
200 MOOS : Scheinb. Gehirnzatälle i. Vexl. ein. eiter. Trommelhöhlenentzünd. etc.

Brechreigung Morgens. Im Laufe des Tages Zunahme des Kopf'schmerzes: kurze Zeit auch Schwindet und Brechneigung; wenig Ohrenschmerz, kein Ausfluss, kein Stuhlgang.

12. Januar. Puls 72, Temp. 30,1 Morgens.

$$
\text { "88, } 730 \text { Abends. }
$$

Zunge rein, kein Appetit, Uebelkeit, Brechneigung, viel Kopfschmers, Schroindel, rechte Pupille etwas enger, kein Ausfluss. Klysma.

Wahrend des Nachmittags Stuhlgang, starke Sehmerzen in der rechten Seite des Kopfes, Empfindlichkeit gegen Druck am Warzenfortsatz. Nach dem Essen Debelkeit und Brechneigung.

13. Januar. Puls 80, Temp. 30,2 Morgens.

$$
\text { "72, "30,2 Abends. }
$$

Heute Morgen Nachlass der Schmerzen; kurze Zeit Schuoindel und Vebelkeit, die sich besonders einstellen, wenn Patientin auf dem Hinterkopf liegt. Austluss Null. Ordin.: 3 Blutigel an den $r$. Warzenfortsatz. Aq. laxativa. -w Abends: kein Stahl. Auch hente ist die rechte Pupille wieder enger, als die linke. Schwindel, Ohrensausen and Kopfschmerz sind geringer. Im Laufe des Nachmittags Lebelkeit und Brechneigung; grosse Mattigkeit.

14. Januar. 8 Stühle. Zustand ohngefähr wie gestern, namentlich die rechte Pupille immer noch enger. Eisblase auf den Kopf.

15. Januar. Puls 84, Temp. 29,6 Morgens.

$$
\text { " } 80, " 30, \hat{3} \text { Abends. }
$$

Drei Stuhlgänge, wenig Schlaf. Schmerz und Gefühl von Brennen iiber und hinter dem rechten Ohr; diesen Morgen eine Zeit lang Uebelkeit, Brechneigung und Schwindel; rechte Pupille enger, als die linke, $\mathrm{Ab}$ und zu Ohrenschmerz. Kein Ausfluss. Im Laufe des Nachmittags heftige Kopfschmerzen, Schwindel und Vebelkeit.

16. Januar, Puls 92, Temp. 30,2 Morgens.

$$
\text { " } 80, \quad 30,5 \text { Abends. }
$$

3 Stühle. Wührend der Nacht heftigo Kopfschmerzen, heute Morgen Uebelkeit und Schwindel. Im Laufe des Tages Nachlass der Schmerzen, kein Ausfuss. Uhr bei Berïhrung.

17. Januar. Puls 88 , Temp. 29,9 Morgens.

$$
\text { "96, "30,3 Abends. }
$$

4 Stühle. Uebelleit und Schwindel in der Nacht und in Laufe des Tages. Starke Schmerzen in der Scheitelgegend gegen Abend.

17. - 21. Januar. Mässiger Kopfsehmerz, dagegen Schwindel, Uebelkeit Brechneigung, Ohrensausen, bedeutende Schwerhörigkeit, kein Ausfluss. Pupillen erst am 21. gleich.

Den 21. Januar Abends untersuchte ich zum ersten Mal die Kranke.

Die Hörweite rechts für mässig laute Sprache 2 Fuss, für eine Uhr von 25 bis 30 Fuss H.-W. 5 Zoll. Die au die Stirne aufgesetzte Stimmgabel wird rechts gehört. Das imnere Ende des Gehörgangs ist grossentheils mit weisslichen (wie die spätere Untersuchung ergab) zusammengeballten Eiter- and Epithelialmassen angefüllt, die sich durch Spritzen nur unvollständig entfernen lassen, so dass die Untersuchung des Trommelfells für diesen Abend unmöglich war. Die Percussion des Kopfes erregt nirgends Schmerz. Beim Katheterísiren hört man ein schwaches trockenes Geräusch, kein Perforationsgeräusch. Patientin fühlt sich subjectiv erleichtert. Die Hörweite beirägt $6 \mathrm{ZOIl}$, resp. 4 Fuss. - Ord: Warmwassereinträuflung. 
MoOS: Scheinb, Gehirnzufălle i. Vexl, eỉn. eiter. Trommelhöhlenentzünd. etc. 201

22. Januar. Puls 76, Temp. 30 Morgens.

" $88, ., \quad 30,4$ Abends.

Zunge rein, kein Appetit; während der Nokht zum ersten Mal hein Schwindel, keine Brechneigung, wenig Kopfschmerz, wenig. Ohrensausen.' Die den Gehörgang ausfiullenden Massen lassen sich heute viel lejchter entfernen. Hörweite nach dem Ausspritzen 9 Zoll. Mussig laute Sprache nur 4 Fuss. Inneres Ende des Gehörgangs stark injicirt und geschwellt; das Trommelfell geröthet und verdickt, Hammartheile unsichtbar; medine ovale Perforation. Nachdem jetzt noch einmal die directe Katheterisirung vorgenommen, bei. welcher ein lebhaftes Perforationsgeräuseh rernommen, and die Kranke das Gefühl hatte als würde ihn , in Inneren des Ohres ein Häutchen weggezogen ", stieg die H.-W. auf $\dot{7}$ Fuss für die Uhr und flüsternde Sprache wurde auf 10 Sohritt deutlich verstanden. Schwindel, Uebelkeit und Erbrechèn, welch schon nach der ersten Katheterisirung weggeblieben waren, 'stellten sich nun auch in "der Folge niemalsimehr ến;" auch keine Ohrensohinerien melir; dagegen für die nächsten 8 Tage noch leichter Kopfschmerz, für die nächsten Wochen ein zeitweilig eintretendes Ohrensauson. Gegen Ende des Monats stellte sich ganz normaler Schlaf ein und konnte P. am 30. Januar zum ersten Male das Bett verlassen, von welcher Zeit an die völlige Wiedergenesung begann. Die weitere örtliche Behandlung tibergehe ich; sie bestand vorzuiglich in Ansspritzen, in der Anwendung einer Zinksolution - 3 Gran auf die Unze.

Am 21." Februar besuchte mich die Kranke in meiner Wohnung. Das Trommelfell war noch dunkel, glanzlos, der Handgxiff, in Säbelform nach rückwärts gehend, war dentlich zu sehen. Eine Perforation weder bei der Inspection des Trommelfells, noch bei der Auscultation zu constatiren. Die Hörweite für eine Uhr von 6 Fuss betrug 2 Fuss und liess für die Sprache nichts mehr zu wïnschen übrig.

Epiterise. Nach dem Aufhören des Ohrenflusses und dem scheinbaren Stillstand der eitrigen Entzïndung stellten sich bei der Kranken Symptome ein, welche mit Recht eine Complikation mit einem ernsten Gehirnleiden befürchten liessen. Diese Symptome bestanden in fast anhaltendem Kopfschmerz, Schwindel, Erbrechen und rechtseitiger Pupillenveränderung. Die letztere wurde wahrscheinlich durch das Ganglion oticum vermittelt; den Schwindel und das Erbrechen dürfen wir um so eher als ein Symptom des gesteigerten intraauriculären Druekes betrachten, als dieselben schon nach der erstmaligen Katheterisirung, obgleich dieselbe nicht einmal vollständig gelang (das Perforationsgeräusch wurde erst bei der zweiten Katheterisirung constatirt), verschwanden, um nicht wiederzukehren. Aber offenbar hatte diese erste Operation schon genügt, einen Theil der in der Trommelhöhle angesammelten Entzïndungsprodukte, durch welche eine vermehrte Belastung der Labyrinthfenster und dadurch der Labyrinthflüssigkeit erzeugt worden war, gegen das Trommelfell, resp. durch seine Perforation in den äusseren Gehörgang: oder gegen die Zellen des Zitzenfortsatzes hinzubewegen. Dafür spricht auch die Angabe der Kranken nach der 
202 MOOS : Soheinb. Gehirnzufalle i. Verl. ein. eiter. Trommelhöhlenentzïind. ete.

zweiten Katheterisirung, sie habe das Gefühl gehabt als würde ihr „im Inneren des Ohres ein Häutchen abgezogen"; vielleicht auch der Umstand, dass sie beim Aufrechtsitzen weniger Schwindel und Uebelkeit empfand, als beim Liegen.

Woit weniger wahrscheinlich, aber immerhin denkbar, scheint mir die Annahme einer durch die Retention der Entzündungsprodukte bedingten Hyperämie des Gehirns und seiner Häute auf der der Trommelhöhlenentzündung entsprechenden Seite. Es spricht namentlich das schnelle Verschwinden der Mehrzahl der beschriebenen Zufälle dagegen. -

Ausfuihrlicher auf die Lebre vom gesteigerten intraauriculären Druck einzugehen, finde ich für heute hier nicht am Platze. 